\title{
NUEVOS OBJETOS Y NUEVAS TÉCNICAS PARA LA EN- SEÑANZA DE LA MATEMÁTICA
}

\author{
Bertha Ivonne Sánchez Luján* \\ Instituto Tecnológico de Ciudad Jiménez, México. \\ ivonnesanchez10@yahoo.com \\ Alberto Camacho Ríos** \\ Instituto Tecnológico de Chihuahua II, México. \\ camachoalberto@hotmail.com
}

Recibido: 27/09/2016 Aceptado: 14/11/2016

\section{Resumen}

En este artículo se presenta un estudio orientado hacia la transposición de un concepto a praxeologías matemáticas. El concepto se desprende de tecnologías externas a las praxeologías, destacándose así un episodio de confrontación de conocimientos prácticos que ocurre al intentar legitimar las diferentes técnicas que de esa inmersión se desprenden. De esa confrontación resultan ambigüedades y rupturas epistemológicas que dan lugar para incorporar tecnologías prácticas para la validación de las técnicas, puesto que el modelo praxeológico no responde al nivel de empirismo resultado de la actividad. El objetivo principal es poner en evidencia la falta de justificación de técnicas externas a la práctica matemática que se transponen para resolver ciertas tareas $\mathrm{T}$ y, además, cómo esa carencia de justificación hace necesarios otros argumentos tecnológicos en la Teoría Antropológica de lo Didáctico para su propia justificación. Palabras clave: Técnica práctica - tecnología teórica - empirema - Institución.

* Profesora del Instituto Tecnológico de Cd. Jiménez. Miembro del Comité Latinoamericano de Matemática Educativa, del Consejo Mexicano de Investigación Educativa. Doctora en Matemática Educativa en el Centro de Investigación en Ciencia Aplicada y Tecnología Avanzada del Instituto Politécnico Nacional.

** Doctorado en Matemática Educativa. Investigador y profesor invitado en diferentes instituciones, de las que destaca el Centro de Investigación en Ciencia Aplicada y Tecnología Avanzada del IPN. Profesor del curso de Ecuaciones en Derivadas Parciales en la Universidad de Chihuahua y Profesor titular en el Instituto Tecnológico de Chihuahua II. 


\section{Abstract:}

This paper presents a study toward the transposition of a concept to mathematics praxeologies. The concept is clear of external technologies to the praxeologies, highlighting and an episode of confrontation of practical knowledge that occurs when attempting to legitimize the different techniques that immersion implies. Ambiguities and epistemological ruptures arise from said confrontation, and give rise to incorporate practical technologies for the validation of the techniques, since the model praxeological does not respond to the level of empiricism outcome of the activity. The main objective is to highlight the lack of justification of techniques that are external to the mathematical practice which are transposed to resolve certain tasks $\mathrm{T}$ and, furthermore, how this lack of justification makes necessary other technological arguments in the Anthropological Theory of Didactics for its own justification.

Keywords: Technical practice - theoretical technology - empirema Institution.

\section{Introducción}

En la Teoría Antropológica de lo Didáctico (TAD) los modelos praxeológicos se asumen organizaciones matemáticas integradas por objetos y técnicas que se consagran en la práctica matemática hacia la resolución de tareas. Desde este enfoque, las actividades de enseñanza-aprendizaje son estructuradas por praxeologías matemáticas cuyos objetos dejan fuera concepciones que dieron origen a la construcción social de los saberes sobre los que descansa actualmente la enseñanza de la matemática. La TAD no acepta la modelación de conceptos fundamentales cuya definición se advierte empírica, por ejemplo: aquellos de variable y función mirados a través del estado del movimiento, percibido este último como variabilidad. La razón es que movimiento y variabilidad no son conceptos que pertenezcan a la teoría matemática y son más bien admitidos en la fenomenología física y los modelos estocásticos.

El uso de recursos de la física y otras disciplinas en la enseñanza de la matemática, ha servido para motivar los tópicos y objetos matemáticos de modo que a través de ellos se puedan interpretar algunos resultados de los problemas prácticos que con esos recursos y otros conceptos se puedan resolver y cuya resolución se exige en los planes y programas de estudio, principalmente en aquellos de las carreras de ingeniería (1). Es así que la introducción de conceptos externos a la práctica matemática deja de lado, eventualmente, la utilidad de las técnicas matemáticas que se despliegan de los teoremas y definiciones, para dar prioridad a otras técnicas empíricas intermediarias que sirven de puente para la resolución de tareas, en tanto establecen ciertas relaciones con las técnicas matemáticas, para desarrollar en el aula los propios teoremas y definiciones que les justifican. 
Revista de la Escuela de Ciencias de la Educación, año 13, nRo. 12, vol. 1, enero a Junio de 2017. PÁGINAS 115-131. ISSN 1851-6297 - ISSN 2362-3349 (EN LiNEA) NuEVOS OBJETOS Y NUEvas técnicas para la enseñanza de la matemática. Bertha Ivonne Sánchez luján - Alberto Camacho Rios

Al menos para la enseñanza, el uso de ese tipo de técnicas para la adquisición del conocimiento ha sido convenido tanto por los autores de textos, los profesores y quienes han diseñado los planes de estudio, sin un cuestionamiento al centro de la teoría matemática que valide su uso.

Ese tipo de convenciones muestra además diferencias en las formas de concebir la enseñanza del saber matemático en las diferentes regiones. No es lo mismo enseñar un concepto en una sociedad altamente desarrollada tecnológicamente, que en otra donde las condiciones sociales de vida son limitadas. No son los mismos planes de estudio, textos y saberes, que se encuentran en juego en ese contraste de sociedades y culturas. En consecuencia, la forma de introducir los conceptos en el aula cambia de una región a otra en un mismo país, incluso de una institución escolar a otra dentro de una misma localidad, y los modos de validar esa introducción también ¿Cómo modelar la actividad de enseñanza aprendizaje bajo esas condiciones desde las praxeologías matemáticas de la TAD?

El objetivo principal de este trabajo es poner en evidencia la falta de justificación de técnicas externas a la práctica matemática que se transponen para resolver ciertas tareas $T$ y, además, cómo esa carencia de justificación hace necesarios otros argumentos tecnológicos en la TAD para su propia justificación.

Cuando se habla de la justificación de las técnicas externas inmersas en las praxeologías, ello refiere un nivel de explicación que el discurso tecnológico otorga a dichas técnicas.

\section{Dos rutas de acceso para la irrupción de conocimientos externos}

El escrito se desarrolla sobre una de las grandes etapas de la TAD cual es la introducción en las praxeologías matemáticas de saberes que se corresponden con disciplinas ajenas a la matemática y su enseñanza. Irrupciones de conocimientos prácticos que se desprenden de técnicas alternativas posibles, y que pertenecen a Instituciones ajenas a las praxeologías, se presentan en la TAD a través de dos rutas de acceso, la primera ocurre durante el episodio de trabajo de la técnica: etapas exploratorias que provocan un desarrollo gradual de la técnica, que a su vez generan técnicas relativamente nuevas en el contexto de la enseñanza y, la segunda, en la dinamización de actividades que se realizan previamente a los momentos de estudio en las organizaciones didácticas (OD), también conocidas como praxeologías didácticas: etapas que llevan sistemáticamente a los estudiantes a la institucionalización del conocimiento. En ambos casos la irrupción desemboca principalmente en el bloque tecnológico teórico $[\theta, \Theta]$-véase más adelante el Esquema 1- trastocando su parte interna. La parte que se trastoca tiene que ver con uno de los objetivos de la tecnología matemática $\theta$, como es la de justificar racionalmente la técnica (Chevallard, 1999, p. 3). La justificación de la técnica, a través del 
discurso tecnológico que se desprende de la tecnología, se presenta entonces ambigua, debido a los estilos de racionalidad que habitan en los espacios institucionales que se ponen a interactuar.

El estilo de racionalidad puesto en juego varía, ello es obvio, en el espacio institucional y, en una institución dada, al filo de la historia de esta institución, de suerte que una racionalidad institucional dada pueda aparecer... poco racional desde la otra institución [2] (Ibid. p. 3).

Lo poco racional de los discursos institucionales puestos en juego se distingue por su nivel de matematización, es decir las diferencias epistemológicas del conocimiento debido a los diferentes campos de referencia involucrados [3] (Moreno, 2006, p. 73), toda vez que la conciliación de esos niveles es lo que se está de por medio.

Para ofrecer un mejor punto de vista alrededor de este fenómeno, se muestra enseguida un breve recorrido de la propuesta de Chevallard para el trabajo de la técnica. Posteriormente se plantean un modelo praxeológico en el que se han introducido conocimientos y técnicas que surgen:

1) De la œuvre matemática $O$ fincada en cierta etapa de la historia y,

2) De instituciones $P$ ajenas a las tecnologías teóricas y a las propias instituciones usuarias dedicadas a la enseñanza de la matemática.

La œuvre $\mathrm{O}$ que refiere Chevallard son documentos históricos, originalmente de naturaleza matemática, de los cuales se rescatan tecnologías externas y con las que se pueden reproducir aquellas técnicas desarrolladas circunstancialmente.

El objetivo es determinar la derivada de una función de grado racional utilizando el método de derivación por incrementos. La idea central es la de contrastar técnicas externas a los modelos praxeológicos con las técnicas matemáticas dominantes y desarrollar con esos argumentos el trabajo de la técnica, de manera que se amplíe la derivación para diferentes funciones como la comentada anteriormente.

En este caso media la práctica matemática en la definición de los objetos en juego, toda vez que en esta actividad, y al menos para la enseñanza, la justificación de la transposición de los conceptos va de la mano con el uso de argumentos empíricos externos a la teoría matemática.

\section{La Teoría Antropológica de lo Didáctico}

Según Chevallard (2007, p. 714), una OM, como la que se describe en el Esquema 1, se compone de los bloques práctico y técnico:

1) El saber-hacer o la praxis $[T, \tau], y$

2) El saber o logos teórico $[\theta, \Theta]$

$$
[T, \tau, \theta, \Theta]
$$

Esquema 1. Modelo praxeológico de Chevallard 
Revista de la Escuela de Ciencias de la Educación, año 13, nRo. 12, vol. 1, enero a Junio de 2017. PÁGINAS 115-131. ISSN 1851-6297 - ISSN 2362-3349 (EN LíNEA) NuEVOS OBJETOS Y NUEvas técnicas para la enseñanza de la matemática. Bertha Ivonne Sánchez luján - Alberto Camacho Rios

En el entorno de una institución usuaria , la actividad escolar $T$ representa las tareas, $\tau$ la técnica o conjunto de realizaciones y procedimientos (el saber-hacer) que permiten abordarlas, $\theta$ la tecnología: objetos matemáticos legitimados para el curso (el saber), como son los teoremas, axiomas y definiciones, que además justifican y hacen inteligible a la técnica, y $\Theta$ la teoría que a su vez justifica a la tecnología. La tecnología $\theta$ se refiere no sólo al estudio de las técnicas que contiene, sino a la manera en que de estas últimas se desprenden: Una tercera función corresponde a un empleo más actual del término tecnología: la función de producción de técnicas [4] (p. 4).

Las praxeologías fundamentales son concebidas como Organizaciones Matemáticas Puntuales (OMP) $[T, \tau, \theta, \Theta]$. Este tipo de organizaciones son comunes y tienen la característica de recrear en el salón de clase una técnica por demás conocida, previamente reconocido su origen, su proceso de construcción social y su colocación en el ambiente escolar. La base de conocimientos matemáticos teóricos que las integran se aprecian en los manuales escolares y en la producción de los encargados de hacer matemática, no obstante, en ambos casos media la transposición institucional de conocimientos. Por su lado, las organizaciones praxeológicas locales (OML) son aquellas que agrupan a una buena cantidad de OMP constituidas cada una alrededor de un único tipo de tareas $T$.

Schneider (2007) ha distinguido dos tipos de praxeologías a través del reconocimiento que hacen los matemáticos de las teorías matemáticas estandarizadas, las cuales están en ruptura con las matemáticas escolares: las praxeologías-modelación que organizan al saber escolar en un primer nivel de justificación matemática y las praxeologías-deducción que representan un nivel principal.

Las praxeologías-modelación son constituidas por objetos pre-construidos de la matemática, las más de las veces sujetos a una misma Institución, y cuya evidencia en el salón de clase no es puesta en duda: (...) "sustitutos de conceptos" a los cuales se les asocian convicciones, "imágenes" que pueden facilitar, o bien obstaculizar, el aprendizaje de los conceptos matemáticos correspondientes" [5] (p. 33).

(...) Como esos objetos no existen todavía como objetos de una teoría y el objetivo es precisamente de constituirles como tales, el discurso que justifica esas técnicas y las rinde inteligibles respecto de la tarea no puede ser teórico, en el sentido en el que lo entienden los matemáticos. Y es esto que hace necesario, me parece, la existencia de un nivel de discurso que Y. Chevallard llama discurso tecnológico [6] (Schneider 2007, p. 33, citada en Gantois, 2012, p. 48)

Las técnicas matemáticas son el vínculo que modela y hace interactuar en las praxeologías a ese tipo de objetos. Siguiendo a Chevallard (1999, p. 
3) Schneider deja ver que los objetos pre-construidos son aquellos embriones de los objetos de la matemática actual: una velocidad que cambia, así como un punto $P(x, y)$ sobre una curva, son objetos pre-construidos. Sin embargo, los ya mencionados variable y función, percibidos a través de la variabilidad, no son, en el sentido de la TAD, embriones de la teoría matemática debido a lo poco racional de la carga empírica que les explica.

\subsection{El trabajo de la técnica}

En Chevallard (2007) la función de la tecnología teórica es: (...) quien, en una institución o persona, reemplaza la función tecnológica: justifica y esclarece la técnica $t$ relacionada con un tipo de tareas $T$, (permitiendo reconstruirla cuando ella es «dada»[7]) (p. 714). La reconstrucción se refiere a la necesidad de retocar la praxeología cuando algún episodio de la técnica lo haga necesario: (...) un episodio de trabajo de la técnica puede conducir a retocar la organización matemática, y eventualmente vivir un nuevo episodio tecnológico -teórico- y en todo caso realizar, aun cuando fuera brevemente, otro episodio de institucionalización [8] (Ibíd. p. 730).

Los episodios de trabajo de la técnica que llevan a retocar la tecnología, refieren la etapa de definir al modelo teórico-matemático de la praxeología. El retoque se realiza durante su organización o estructuración y en particular durante el trabajo de la técnica. El objetivo en esta etapa es mejorar la técnica y dejarla lo más eficaz y confiable posible, incorporando a esta última técnicas alternativas. Con ello se exigen modificaciones a la tecnología que la llevan a acrecentar el dominio y control con los que cuenta.

El ejemplo de una obra $O$ de la que se rescata una técnica $\tau_{j}$ y la tecnología $\theta_{i}$ de la que se desprende, para calcular el valor de $x$ con el cual el perímetro $2 p$ de un rectángulo deviene máximo, se muestra en Chevallard, (1999) de la siguiente manera:

La técnica elemental utilizada en ausencia del cálculo infinitesimal se funda sobre el resultado siguiente: si $x_{1}, x_{2}, \ldots, x_{n}$ son reales $\geq 0$, siendo la suma constante, igual a a, entonces el producto $x_{1} x_{2} \ldots x_{n}$ es máximo luego que $x_{1}=$ $x_{2}=\ldots=x_{n}=a / n$. Luego el área de un perímetro $2 p$, que se escribe como $x y$, con $x+y=p$, es máxima luego que $x=y=p / 2$, es decir luego que el rectángulo es un cuadrado [9] (p. 14).

Con la irrupción en la praxeología de la técnica y la tecnología, así recuperadas, ocurre una transposición de nuevos objetos que nutren a la tecnología original y la transforman en una tecnología mixta. Se trabaja en esa etapa con embriones del conocimiento (Ibíd. p. 3), desarrollados y utilizados a lo largo de varios siglos que toman la forma de OMP, y que se constituyen alrededor de un único tipo de tarea. Al menos para el caso citado, Chevallard no aclara la justificación de la transposición de nuevo conocimiento, ni cómo es el resultado de la nueva tecnología mixta, tampoco asume el control de las rupturas 
Revista de la Escuela de Ciencias de la Educación, año 13, nRo. 12, vol. 1, enero a Junio de 2017. PÁGINAS 115-131. ISSN 1851-6297 - ISSN 2362-3349 (EN LíNEA) NuEVOS OBJETOS Y NUEvas técnicas para la enseñanza de la matemática. Bertha Ivonne Sánchez luján - Alberto Camacho Rios

epistemológicas que se producen.

Por la naturaleza de los documentos donde se rescatan las técnicas, es decir la obra matemática $O$, no hay afectación en las decisiones matemáticas y de práctica matemática a que da lugar la tecnología dominante para operar, accionar y legitimar sus propias funciones.

El estudio de las obras $O$ tiene como finalidad encontrar una respuesta que, incluso, haya sido producida con antelación en instituciones externas a la didáctica para reconstruirla o transponerla a la institución usuaria en estudio, en tanto con ella resolver las tareas $T$ que ahí se impongan.

Estudiar una cuestión del tipo $\tau_{T}$, donde $T$ es un cierto tipo de tareas, conduce -como un principio del mundo científico- a crear una respuesta, es decir a elaborar una organización praxeológica $O=[T / \tau / \theta / \Theta]$ inédita. Más, en el mundo ordinario de la skholê, estudiar una cuestión, es, casi siempre, recrear, para sí mismo y sus compañeros de estudio, una respuesta O ya producida en alguna otra institución. Estudiar, es luego estudiar una respuesta (en un sentido enérgico) tenida por válida. Es estudiar una œuvreque ha existido siempre en la sociedad, para reconstruirla, transponerla en la institución que servirá de hábitat al estudio. El paso del estudio de una cuestión al estudio de una respuesta -de una œuvre- no va sin algunas modificaciones (incluso) en la noción misma de estudio [10] (p. 14).

La skholê descrita por Chevallard, representa la escuela. No obstante, la reconstrucción de œuvres $\mathrm{O}$ que se puedan transponer a una institución escolar, en este caso a las praxeologías matemáticas que habitan en esas instituciones, requieren de justificaciones que no se perciben inmediatas alrededor de la transposición. No es suficiente saber que en $O$ existen nuevas técnicas pertinentes, ni tampoco de cuales teoremas se desprenden. Es necesario conocer más al respecto.

\subsection{El modelo praxeológico extendido de Castela y Romo Vázquez. La emergencia de la tecnología práctica $\theta^{p}$}

Al centro del modelo praxeológico, Castela (2008) y Castela y Romo Vázquez (2011) han distinguido seis funciones posibles de la tecnología $\theta$ que justifican las técnicas que de ella se desprenden: describir, facilitar, motivar, evaluar, validar y explicar: Según las autoras, entre los saberes que se contemplan en las seis funciones algunos se determinan en el marco de una teoría matemática y otros surgen de la práctica matemática, quien provee su validación en un contexto empírico. La validación de las funciones citadas debe ir acorde a la situación de la teoría matemática que cobija a la tecnología, y desarrollarse en forma de hipótesis que surgen de los teoremas que resultan de la propia tecnología. Al analizar el modelo praxeológico que involucra la solución de ecuaciones diferenciales lineales a través de la Transformada de 
Laplace, en el contexto de la enseñanza de la Ingeniería —en Francia - principalmente en el curso AU2 de Automática(segundo curso de Automática de la carrera de Ingeniería donde las autoras realizaron el estudio), encontraron que ciertos elementos de saberes que corresponden a la tecnología teórica $\theta^{\text {th }}$ , desembocan de procesos no teóricos [11].

Estos últimos procesos son determinados y validados por las instituciones usuarias $I_{u}$ de la matemática que no se pueden disociar de los modelos praxeológicos por su situación en la enseñanza de la matemática.

Particularmente, el discurso en el cual la integral de la función impulso Delta de Dirac vale uno en , la validación que aparece en el Anexo del curso AU2, es vista como una explicación necesaria al centro de las argumentaciones formales, más no conforme con las normas de la teoría matemática y si, en los más de los casos, al contexto fenomenológico de la física. La explicación se refiere al estado en que se encuentra la validación de las argumentaciones en su proceso de matematización. La situación es incómoda puesto que la tecnología $\theta^{\text {th }}$ que valida las técnicas en el juego de la praxeología matemática deviene ambigua, poco tolerante, y con desviaciones hacia la justificación de conocimientos prácticos que emergen de la fenomenología física.

Ante la pérdida de dominio por parte de la tecnología teórica $\theta^{\text {th }}$ en la legitimación de las funciones de la técnica, estas últimas deben ser incluidas en una tecnología práctica intermediaria $\theta^{p}$ que las justifique, toda vez que validadas por la institución usuaria $I_{u}$.

La brecha que se abre entre los conocimientos contenidos en $\theta^{p}$ y $\theta^{\text {th }}$, en el modelo extendido propuesto en el Esquema 2, hace que la posición de $\theta^{p}$ sea incierta por la teoría matemática en la que se encuentra ubicada la praxeología. Ante esto último, Castela y Romo Vázquez (2011) proponen un intento de justificación de los conocimientos prácticos: (...) una épure praxeológica: (...) donde el nivel práctico debe ser forjado por la institución usuaria, tomando apoyo sobre la diversidad de temas que en esa institución pondrán en práctica la técnica [12] (p. 17).

Una épure praxeológica es una OM que se encuentra en proceso de legitimación debido a que contiene conocimientos empíricos. El modelo praxeológico extendido que incluye ambas tecnologías es propuesto en el Esquema 2.

$$
\left[\begin{array}{cccc}
T, & \tau, & \theta^{\text {th }} & \Theta \\
& \theta^{p} &
\end{array}\right] \begin{aligned}
& \leftarrow P(M) \\
& \leftarrow I_{u}
\end{aligned}
$$

Esquema 2. Modelo praxeológico extendido propuesto por Castela y Romo Vázquez (2011), en el que se incluye una tecnología práctica intermediaria $\theta^{p}$. 
Revista de la Escuela de Ciencias de la Educación, año 13, nRo. 12, vol. 1, enero a Junio de 2017. PÁGINAS 115-131. ISSN 1851-6297 - ISSN 2362-3349 (EN LiNEA) NuEVOS OBJETOS Y NUEvas técnicas para la enseñanza de la matemática. Bertha Ivonne Sánchez luján - Alberto Camacho Rios

Sin embargo, en el caso donde la tecnología práctica se determina a partir de actividades ajenas a las $\mathrm{OM}$, como sucede con aquellas contenidas en las prácticas sociales que constituyen a las Instituciones $P$, ajenas a las instituciones educativas, la brecha que se abre entre las tecnologías $\theta^{p}$ y $\theta^{\text {th }}$ es semejante a los procesos de construcción de conocimiento matemático que han ocurrido a lo largo de la historia, incluso en ciertos casos extremos $\theta^{\text {th }}$ se puede ver como el límite al que tiende $\theta^{p}$. Bajo esas circunstancias, las técnicas son rescatadas de las tecnologías prácticas $\theta^{p}$ y el intento de legitimación al centro del modelo praxeológico ocurre desde la Institución $P$, ajena -en algunos casos- a las cuestiones de enseñanza del conocimiento, cuyo nivel de matematización suele estar alejado de la propia legitimación de las técnicas que se desprenden de $\theta^{\text {th }}$, presentándose la ambigüedad ya citada.

\section{Irrupción transpositiva de conocimientos externos a los modelos praxeológicos}

En la sección 4.1 se presenta el caso de una actividad comprendida en una OMP, en la que se propone el uso de la técnica matemática $\tau_{m}$ de derivación por incrementos, también conocida como regla de los cuatro pasos [13], para derivar funciones con exponentes fraccionarios de la forma: $f(x)=\frac{1}{\sqrt[n]{x}}$, para $n$ entero natural (Engler\& Camacho, 2012; Engler, 2014, p. 139). Importa confrontar conocimientos externos al modelo praxeológico (Camacho \& Romo-Vázquez, 2015) dominante, debido a que, como se verá, este último es una OM incompleta en el sentido de Fonseca, Boch y Gascón (2007) que hace necesario el trabajo de la técnica. En ese episodio se recupera de la obra matemática $O$ un objeto pre-construido en el sentido de Schneider (2007), que integra una técnica que se complementa bien con la técnica matemática, más su inclusión, y sobre todo los elementos que la configuran, no se corresponden con las normas que definen a la tecnología teórica dominante. El ejemplo encubre una comunidad de expertos en ciencias de la tierra utilizando conocimientos prácticos en sus actividades personales.

\subsection{Una técnica que se desprende de la tecnología dominante}

La praxeología para la derivación de funciones $f(x)$ a partir de la definición de derivada -al menos en el sistema de enseñanza superior mexicanotoma una estructura semejante a la siguiente:

\section{Organización Matemática Dominante}

- Tipo de tareas T: Derivar por incrementos la función $f(x)=\frac{1}{\sqrt[n]{x}}$, para $n$ entero positivo, aplicando la definición de derivada.Técnica dominante $\tau_{m}$ : Derivación por incrementos: 
- Procedimiento: Regla de los cuatro pasos:

$\tau_{1}$ : Se incrementa $f(x) \rightarrow f(x+\Delta x)$

$\tau_{2}:$ Se establece la diferencia: $f(x)-f(x+\Delta x)$

$\tau_{3}:$ Se dividen ambos miembros por $\Delta x$

$\mathrm{\tau}_{4}:$ Se aplica $\lim _{\Delta x \rightarrow 0} \frac{f(x+\Delta x)-f(x)}{\Delta x}$

Siendo: $f^{\prime}(x)=\lim _{\Delta x \rightarrow 0} \frac{f(x+\Delta x)-f(x)}{\Delta x}$

- Tecnología $\theta^{\text {th: }}$ La definición de la tecnología son los cuatro pasos

enunciados y resumidos en la expresión: $f^{\prime}(x)=\lim _{\Delta x \rightarrow 0} \frac{f(x+\Delta x)-f(x)}{\Delta x}$,

Se encuentra aquí un discurso tecnológico estandarizado que involucra técnicas matemáticas que la fortalecen y que, efectivamente, se desprenden de la tecnología dominante.

Al analizar en los textos de cálculo diferencial los tipos de funciones cuya derivada se determina con la regla de los cuatro pasos, se verá que la mayoría están preparadas para funciones polinómicas elementales -en algunos casos con radicales- que no sobrepasan el grado dos o tres. De esa manera se logra que la técnica matemática domine las tareas propuestas, sin que simultáneamente ocurra un cuestionamiento tecnológico (Fonseca, et. al, 2007) dada la propia limitación de la técnica y debido a la comodidad de su uso con las funciones mencionadas. En este caso la tecnología dominante incide directamente sobre la técnica matemática, disminuyendo su potencial algorítmico y algebraico.

La técnica matemática $\tau_{m}$ que se relaciona con la praxeología, y que se desprende de la definición de derivada (1) incorporada en $\theta^{\text {th }}$ :

$$
f^{\prime}(x)=\lim _{\Delta x \rightarrow 0} \frac{f(x+\Delta x)-f(x)}{\Delta x}-\text { Si este límite existe- } \ldots(1)
$$

es por sí misma ineficaz para resolver la tarea $T$ por los procedimientos algebraicos que involucra y debido a lo exagerado de los índices de los radicales que surgen en el proceso de cálculo de la derivada. Si por ejemplo, en un caso extremo, $\mathrm{n}=5$ se supone para $f(x)=\frac{1}{\sqrt[5]{x}}$, la diferencia: $f(x+\Delta x)-f(x)=\frac{1}{\sqrt[5]{x+\Delta x}}-\frac{1}{\sqrt[5]{x}}$, y en consecuencia el procedimiento algebraico para el paso al límite, quedan por demás incómodos y algebraicamente fortalecidos.

Para desarrollar el trabajo de la técnica en la praxeología dominante, pudiera pensarse en utilizar otra técnica alternativa $\tau_{p}$ como el teorema del binomio de Newton. Esta última fue comúnmente utilizada para matematizar 
Revista de la Escuela de Ciencias de la Educación, año 13, nRo. 12, vol. 1, enero a junio de 2017. PÁGINAS 115-131. ISSN 1851-6297 - ISSN 2362-3349 (EN LiNEA) NuEVOS OBJETOS Y NUEvas técnicas para la enseñanza de la matemática. Bertha Ivonne Sánchez luján - Alberto Camacho Rios

problemas de variación en la física, así como cuestiones de geometrización por comunidades de ingenieros geógrafos, topógrafos y astrónomos, a lo largo del siglo XVIII y hasta principios del siglo XX, y fundamentalmente en la enseñanza de ese concepto. Una expresión algebraica y variacional del teorema se presenta en (2):

$$
f(x+\Delta x)=f(x)+A h+B h^{2}+e t c ., \square \text { Donde: } h=\Delta x \square \ldots
$$

La serie fue legitimada por Abel en 1826 desarrollando para ello una demostración rigurosa que aparece en Le Journal de Crelle. Por su lado Lagrange, en la Théorie des fonctions analytiques, sugería la expresión (2) como:

$$
f(x+i)=f_{x}+p i+q i^{2}+e t c
$$

Donde $p, q, r$, etc., representan las funciones derivadas de. Esa última serie fue esencial para la construcción del análisis algebraico que permeó los siglos XVIII y XIX. En tal sentido es posible afirmar que el origen de la técnica $\tau_{p}$ aparece en la œuvre matemática $O$ hasta aquí citada.

Por si mismo, el teorema del binomio tiene condiciones suficientes para mejorar la derivación por incrementos de funciones que no es posible atender con la técnica matemática dominante.

De (2) se determina la derivada para el caso de funciones analíticas como se aprecia en (4), la cual es una representación de la tecnología intermediaria $\theta^{p}$ :

$$
\begin{gathered}
\left.f^{\prime}(x)=\lim _{\Delta x \rightarrow 0} \frac{f(x+\Delta x)-f(x)}{\Delta x}-B \Delta x-C(\Delta x)^{2}+e t c .\right]=A+\lim _{\Delta x \rightarrow 0} \varepsilon(\Delta x), \text { donde } \\
\varepsilon(\Delta x)=-B \Delta x-C(\Delta x)^{2}+e t c . \text { Siendo, } \lim _{\Delta x \rightarrow 0} \varepsilon(\Delta x)=0 \ldots(4)
\end{gathered}
$$

Utilizando el procedimiento de los cuatro pasos y la técnica $\tau_{p}$ del teorema del binomio citada en (2), se puede determinar la derivada de la función analítica propuesta inicialmente, es decir:

$\mathrm{\tau}_{1}$ : Se incrementa $f(x+\Delta x)=(x+\Delta x)^{-\frac{1}{n}}=x^{-\frac{1}{n}}-\frac{1}{n} x^{-\frac{1}{n}-1} \Delta x+\frac{\frac{1}{n}\left(\frac{1}{n}+1\right) x^{-\frac{1-2}{n}}(\Delta x)^{2}}{2}+\ldots$

$\tau_{2}$ : Se establece la diferencia: $f(x+\Delta x)-f(x)=x^{-\frac{1}{n}}-\frac{1}{n} x^{-\frac{1-1}{n}} \Delta x+\frac{\frac{1}{n}\left(\frac{1}{n}+1\right) x^{-\frac{1-2}{n}}(\Delta x)^{2}}{2}+\ldots-x^{-\frac{1}{n}}$

$\tau_{3}:$ Se dividen ambos miembros por $\Delta x$ :

$$
\begin{aligned}
& \frac{f(x+\Delta x)-f(x)}{\Delta x}=-\frac{1}{n} x^{-\frac{1}{n}-1}+\varepsilon(\Delta x) \\
& \text { Donde: } \varepsilon(\Delta x)=\frac{\frac{1}{n}\left(\frac{1}{n}+1\right) x^{-\frac{1}{n}-2} \Delta x}{2}+\ldots
\end{aligned}
$$

$\tau_{4}$ : Se aplica (3) como: $\lim _{\Delta x \rightarrow 0} \frac{f(x+\Delta x)-f(x)}{\Delta x}+\lim _{\Delta x \rightarrow 0} \varepsilon(\Delta x)$ 


$$
\lim _{\Delta x \rightarrow 0} \frac{f(x+\Delta x)-f(x)}{\Delta x}=-\frac{1}{n} x^{-\frac{1-1}{n}-1}+\frac{\frac{1}{n}\left(\frac{1}{n}+1\right) x^{-\frac{1}{n}-2} \lim _{\Delta x \rightarrow 0}(\Delta x)}{2}+\lim _{\Delta x \rightarrow 0} \varepsilon(\Delta x)
$$

Siendo: $f^{\prime}(x)=\lim _{\Delta x \rightarrow 0} \frac{f(x+\Delta x)-f(x)}{\Delta x}+\lim _{\Delta x \rightarrow 0} \varepsilon(x)$ :

$$
f^{\prime}(x)=-\frac{1}{n} x^{-\frac{1}{n}-1}
$$

La funciones de la tecnología intermediaria $\theta^{p}$ que valida la técnica de los cuatro pasos empleada, se desprenden directamente de la expresión (4). La justificación de la técnica se puede formular a través de las seis funciones tecnológicas propuestas por Castela (2008) y Castela y Romo Vázquez (2011), tal y como se plantean enseguida.

\section{Validar la técnica}

La técnica $\tau$ es validada por la tecnología práctica $\theta^{p}$ Dado cualquier número $x$ para el cual este límite exista, asignamos a $x$ el número $f(x)$. De modo que podamos considerar $A=\frac{\lim _{\Delta x \rightarrow 0}}{[x}\left[\frac{f(x+h)-f(x)}{h}-B h-C h^{2}+\right.$ etc. $] \quad$ o bien: $f^{\prime}(x)=\lim _{\Delta x \rightarrow 0} \frac{f(x+\Delta x)-f(x)}{\Delta x}+\lim _{\Delta x \rightarrow 0} \varepsilon(\Delta x)$, como una nueva función, llamada derivada de $f$ y definida por medio de la ecuación anterior.

\section{Describir la técnica}

La descripción de $\tau$ se desprende de (4), para lo cual se siguen los siguientes cuatro pasos:

$\tau_{1}$ : En la función $\mathrm{f}(\mathrm{x})$ cambiar $x$ como $x+\Delta x$,

$\mathrm{\tau}_{2}$ : Restar $\mathbf{f}(\mathbf{x})$ de,$f(x+\Delta x)=f(x)+A \Delta x+B(\Delta x)^{2}+e t c .=f(x)+A \Delta x+\varepsilon(\Delta x)$

$\tau_{3}$ : Dividir el resto por $\Delta x y$,

$\tau_{4}$ : Calcular el límite al cual tiende el cociente cuando $\Delta x$ tiende a cero, si es que este límite existe.

\section{Facilitar el empleo de la técnica}

Se eligen cualquier tipo de funciones analíticas. Por ejemplo aquellas de la forma: $f(x)=a_{a^{n}} x^{n}+a_{n-1} x_{a}^{n-1}+\ldots+a_{1} x+a_{0}$ donde $n$ es cualquier real, 0 bien por ejemplo: $f(x)=\frac{a^{n}}{x^{10}}$ y $f(x)=\frac{a}{\sqrt[5]{x}}$.

\section{Motivar la técnica}

La técnica $\tau$ es importante porque permite determinar la derivada de cualquier función analítica $\mathrm{f}(\mathrm{x})$ haciendo uso de la definición de derivada.

\section{Explicar la eficiencia}

La técnica $\tau$ lleva a determinar con eficiencia las reglas fundamentales de derivación, así como la derivada de cualquier función vista previamente en el curso, por ejemplo la derivada del producto de dos funciones, el cociente, etc. 


\section{Evaluar la técnica}

La definición de derivada expuesta en (4) se adecúa bien para determinar las derivadas de funciones elementales como las que se mostraron anteriormente, es fácil probar que cuando las funciones por derivar dejan de ser de ese tipo, por ejemplo funciones no desarrollables en serie de potencias, la técnica se vuelve obsoleta.

En el caso citado no se puede hablar de una tecnología práctica que nutra a la tecnología teórica que se muestra en (1) y se determine así una tecnología mixta, debido al rigor del discurso que se desprende del bloque tecnológico que justifica la praxeología dominante, sino más bien de una tecnología práctica intermediaria $\theta^{p}$ que justifica el uso de la técnica del teorema del binomio. La irrupción de conocimientos jerarquizada por la Institución $O$ en la praxeología, se aprecia enseguida en el Esquema 3.

$$
\begin{gathered}
{\left[\theta^{p}\right] \leftarrow 0} \\
{\left[\begin{array}{c}
\downarrow \\
T, \begin{array}{l}
\tau_{p} \rightarrow \\
\tau_{\text {th }} \leftarrow
\end{array} \theta^{\text {th }}, \Theta
\end{array}\right] \leftarrow I_{u}}
\end{gathered}
$$

Esquema 3. La tecnología intermediaria $\theta^{p}$ surge de la œuvre $O$ ajena a la enseñanza de la derivada y provee de técnicas $\tau_{p}$ para desarrollar el episodio del trabajo de la técnica en la praxeología dominante.

Con la inclusión de la técnica externa del teorema del binomio de Newton, al centro del procedimiento de la Regla de los Cuatro Pasos, se mejora considerablemente la resolución de tareas, incorporando incluso aquellas que la técnica dominante no resuelve. Es así que esta última se dinamiza a través de los argumentos variacionales del teorema, dando por resultado una técnica híbrida útil en el salón de clases, no obstante ¿Cómo conciliar los niveles de matematización involucrados a través de ambas instituciones, en este caso la $œ u v r e ~ O$ y las instituciones usuarias del conocimiento $I_{u}$ ?

\section{Algunos resultados}

La técnica $\tau_{p}$ del teorema del binomio dinamiza los procedimientos de orden algebraico contenidos en la técnica matemática $\tau_{m}$ que se desprende de la tecnología teórica $\theta^{\text {th }} \mathrm{y}$ ayuda a resolver los problemas como el que se puso en evidencia, donde los índices de los radicales son difíciles de manipular con los procedimientos algebraicos tradicionales, ampliando así el campo de tareas $T_{i}$ que se pueden resolver.

No obstante las diferencias entre ambas, la técnica $\tau_{p}$ se impone en el aula sobre la técnica matemática, resultando así una técnica híbrida que contiene 
conocimientos aproximados, alterando así el bloque técnico-práctico $[T, \tau]$ y, en consecuencia, al bloque tecnológico teórico $[\theta, \Theta]$. Esa aproximación del conocimiento que resulta, confirma un dominio más amplio de la praxeología que da para ponerlo a prueba en el salón de clases. Sin embargo, los modos de validación de los conceptos aproximados que resultan, no van del lado de las exigencias de la tecnología teórica.

En el caso de las praxeologías que atendieron Castela y Romo Vázquez (2011), la tecnología práctica $\theta^{p}$ surge de la práctica matemática que se asocia a la fenomenología física. Esta posición mixta convencional que incorpora ambas tecnologías, establece una épure praxeológica de la que habría que eliminar las coyunturas de la práctica matemática en un contexto donde la comunidad de expertos $P(M)$ que se dedican a hacer matemática, lleven los conocimientos prácticos o empíricos a una posición más avanzada de matematización, principalmente al nivel de la teoría matemática en la que se circunscribe la propia praxeología. Es la propuesta del modelo praxeológico extendido de Castela y Romo Vázquez (2011) una evolución importante que inserta el conocimiento empírico al centro del modelo praxeológico de Chevallard.

\section{Conclusiones}

En la situación donde el empirismo de las técnicas surge de actividades no ajenas a una teoría matemática, al seno de la práctica matemática misma, validada por una tecnología práctica $\theta^{p}$, pero alejada de la tecnología teórica límite que le sirve de referencia, la épure praxeológica que se determina establece una amplia brecha epistemológica con la inmersión de conocimientos aproximados, como resulta ser el caso presentado de la derivada. En ese punto, la justificación de las técnicas se fincan a partir de definiciones alejadas todavía de la teoría matemática, y son vistas como vestigios o embriones de las técnicas dominantes (Chevallard, 1999. p. 3). Las justificaciones se ubican en la œuvre matemática $O$ de los conceptos involucrados y su validación al centro de las praxeologías depende de la flexibilidad de las tecnologías dominantes y de las instituciones usuarias $I_{u}$. De darse esa flexibilidad en las tecnologías teóricas, se reducirían los niveles de racionalidad en el salón de clases por la inclusión de las tecnologías prácticas, pero mejoraría sustancialmente el discurso matemático de los conceptos en juego.

\section{Notas Bibliográficas}

(1) Los problemas de ingeniería son sugeridos para ser resueltos en los planes de estudio de las carreras de ingeniería de los sistemas universitario y tecnológico mexicano.

(2) Le style de rationalité mis en jeu varie bien entendu dans l'espace institutionnel, et, en une institution donnée, au fil de l'histoire de cette institution, de sorte qu'une 
Revista de la Escuela de Ciencias de la Educación, año 13, nRo. 12, vol. 1, enero a junio de 2017. PÁGINAS 115-131. ISSN 1851-6297 - ISSN 2362-3349 (EN LiNEA) NuEVOS OBJETOS Y NUEvas técnicas para la enseñanza de la matemática. Bertha Ivonne Sánchez luján - Alberto Camacho Rios

rationalité institutionnelle donnée pourra apparaître... peu rationnelle depuis telle autre institution.

(3) Según Moreno, un campo de referencia es un campo complejo conformado con símbolos que dan significado a aquellos términos que forman parte de un mismo sistema: "En las matemáticas, por ejemplo, un término cobra sentido en términos de los demás que forman parte del mismo sistema. Nociones como "derivada" e "integral”, requieren, entre otras, de las nociones de función y aproximación para alcanzar un cierto nivel de significación" (p. 75). Moreno asume la definición de campo de referencia al nivel de la cognición de los sujetos: estudiantes, profesores, etcétera, no obstante Chevallard contempla los estilos de racionalidad desde una cognición institucional que, si bien no trata a los sujetos en lo particular, si los involucra.

(4) Enfin une troisième fonction correspond à un emploi plus actuel du terme de technologie: la fonction de production de techniques.

(5) (...) "substituts de concepts" aux- quells sont associées des convictions, des "images" qui peuvent soit faciliter, soit entraver l'apprentissage des concepts mathématiques correspondants

(6) (...) Commeces objets n'existent pas encore comme objets d'une théorie et que le but est précisément de les constituer commetels, le discours qui justifie ces techniques et les rend intelligibles eu égard à la tâche visée ne peut être théorique, au sens où l'entendraient des mathématiciens. Et c'est ce qui rend nécessaire, me semble-t-il, l'existence d'un niveau de discours qu'Y. Chevallard appellee discours technologique.

(7) (...) qui, dans une institution ou pour une personne, remplit la function technologique - justifier, éclairer la technique $\tau$ relative au type de tâches $T$, voire permettre de l'engendrer (ou de la reconstruire, quandelleest « donnée ».

(8) (...) un épisode de travail de la technique peut conduire à retoucher l'organisation mathématiquemise en place, et donc éventuellement à vivre un nouvel episode technologico - théorique - et en tout cas à envisager, si bref soit-il, un autre episode d'institutionnalisation.

(9) La technique élémentaire utilisée en l'absence de calcul infinitésimal se fondait sur le résultat technologique suivant: si $x_{1}, x_{2}, \ldots, x_{n}$ sont des réels $\geq 0$ dont la somme est constante, égale à $a$, alors le produit $x_{1} x_{2} \ldots x_{n}$ est maximal lorsque $x_{1}=x_{2}=\ldots=x_{n}=$ $a / n$. Ainsi l'aire d'un rectangle de périmètre $2 p$, qui s'écrit $x y$, avec $x+y=p$, est-elle maximale lorsque $x=y=p / 2$, c'est-à-dire lorsque rectangle est un carré.

(10) Étudier une question du type $\tau_{T}$, où $T$ est un certain type de tâches, cela conduit - comme il en va en príncipe dans le monde savant - à créer une réponse, c'est- à-dire à élaborer une organisation praxéologique $O=[T / \tau / \theta / \Theta]$ inédite. Mais, dans le monde ordinaire de la skholê, étudier une question, c'est, presque toujours, recréer, poursoi et ses compagnons d'étude, une réponse $O$ déjà produite en quelque autre institution. Étudier, c'est donc étudier une réponse (au sens fort) tenue pour valable. C'est étudier une œuvre existant ailleurs dans la société, pour la reconstruire, la transposer dans l'institution qui sert d'habitat à l'étude. Le passage 
de l'étude d'une question à l'étude d'une réponse - d'une œuvre - ne va pas sans quelques modifications dans la notion même d'étude.

(11) Castela y Romo Vázquez consideran $\theta$ como $\theta^{\text {th }}$ (th: théorie) significando así la naturaleza teórica de la tecnología.

(12) (...) une épure praxéologique dont le niveau pratique devra être forgé par l'institution utilisatrice en prenant notamment appui sur les communautés des sujets qui dans cette institution auront à mettre en œuvre la technique.

(13) En los niveles de enseñanza medio superior y superior mexicanos, en los cursos de cálculo diferencial, la regla de los cuatro pasos también es reconocida como método de derivación por incrementos.

\section{Referencias bibliográficas}

- Camacho, A. \& Romo-Vázquez, A. (2015). Déconstruction-construction d'un concept mathématique. (Ed.) Pluralités culturelles et universalité des mathématiques: enjeux et perspectives pour leur enseignement et In Theis L.

- Castela, C. (2008). La noción de praxeología: un instrumento de la Teoría Antropológica de lo Didáctico posiblemente útil para la Socioepistemología. En P. Lestón (Ed.) Acta Latinoamericana de Matemática Educativa Vol. 22 (pp. 1195-1206). México: Comité Latinoamericano de Matemática Educativa. Disponible en http://clame. org.mx/documentos/alme22.pdf

- Castela, C. \& Romo Vázquez, A. (2011). Des mathématiques a l’Automatique: Étude des effets de transposition sur la transformée de Laplace dans la formation des ingénieurs. Recherches en Didactique des Mathématiques, 31(1), 79-130.

- Chevallard, Y. (1999). L'analyse des pratiques enseignantes en théorie anthropologique du didactique. Consultado el 20 de abril de 2013, En:

- http://yves.chevallard.free.fr/spip/spip/IMG/pdf/Analyse_des_pratiques_enseignantes.pdf

- Chevallard, Y. (2007). Passé et présent de la Théorie Anthropologique du Didactique. En Ruíz-Higueras, L., Estepa, A. \& García, F. J. (Eds.). Sociedad, Escuela y Matemáticas. Aportaciones a la Teoría Antropológica de lo Didáctico (pp. 705-746). España: Servicio de Publicaciones de la Universidad de Jaén.

- Engler, A. (2014). Construcción de la derivada a través de dinamizar la regla de los cuatro pasos. Aproximación socioepistemológica. México: Centro de Investigación en Ciencia Aplicada y Tecnología Avanzada del Instituto Politécnico Nacional. Tesis de doctorado publicada parcialmente.

- Engler, A. \& Camacho, A. (2012). Construcción de la derivada. Dinamización de la regla de los cuatro pasos. Berlín: Editorial Académica Española. LAMBERT Academic Publishing. GmbH\& Co. KG.

- Fonseca, C., Boch, M. \&Gascón, J. (2007). El momento del trabajo de la técnica en la completación de organizaciones matemáticas: el caso de la "Regla de Ruffini". En Ruíz-Higueras, L., Estepa A. \& García, F. J (Eds.). Sociedad, Escuela y Matemáticas. Aportaciones a la Teoría Antropológica de lo Didáctico (pp.139-157). España: 


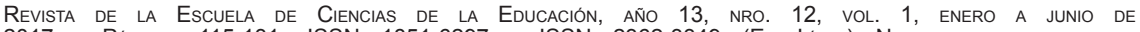
2017. PÁGINAS 115-131. ISSN 1851-6297 - ISSN 2362-3349 (EN LiNEA) NuEVOS OBJETOS Y NUEvas técnicas para la enseñanza de la matemática. Bertha Ivonne Sánchez luján - Alberto Camacho Ríos

Servicio de Publicaciones de la Universidad de Jaén.

- Gantois, J-Y. (2012). Un milieu graphique-cinématique pour l’apprentissage des dérivées dans une paraxéologie "modélisation": potentialités el limites. Thèse de doctorat en didactique des mathématiques, Faculté des Sciences, Université de Liège.

- Moreno, A. L. (2006). Nociones matemáticas, constitución del símbolo y evolución del campo de referencia. En E. Filloy (Autor y Compilador). Matemática Educativa, treinta años: una mirada fugaz, una mirada externa y comprensiva, una mirada actual (pp. 69-83). México: Aula XXI/Santillana.

- Schneider, M. (2007). Entre recherche et développement: quel choix de valeurs pour l'ingénierie curriculaire? Intervention aux journées mathématiques organisées par I'INRP, 12 juin 2007, http://www.inrp.fr/. 